\title{
CHARACTERIZATION OF ELECTRO-RHEOLOGCIAL FLUIDS UNDER HIGH SHEAR RATE IN PARALLEL DUCTS
}

\author{
X. W. ZHANG ${ }^{\dagger}$, C. B. ZHANG ${ }^{2}$, T. X. YU ${ }^{3}$ \\ Department of Mechanical Engineering, Hong Kong University of Science and Technology, \\ Clear Water Bay, Hong Kong, P. R. CHINA, \\ zhangxw@ust.hk,zcxaa@ust.hk,metxyu@ust.hk
}

W. J. WEN ${ }^{4}$

Department of Physics, Hong Kong University of Science and Technology

Clear Water Bay, Hong Kong, P. R. CHINA,

phwen@ust.hk

Received 15 June 2008

Revised 23 June 2008

\begin{abstract}
Electro-rheological (ER) fluid is a smart suspension which can be changed promptly from Newtonian to Bingham plastic material when subjected to a high-intensity electric field. This property of ER fluid makes it possible to be applied in adaptive energy absorbers. As the impact velocity encountered in applications could be very large, it is necessary to characterize the ERF under high shear rate. In this study, a capillary rheo-meter with parallel duct was designed and manufactured which is capable of producing a shear rate as high as 5000(1/s). Two giant ER fluids with mass concentration $\mathrm{C}=51 \%$ and $44.5 \%$ and a commercial density-matched ER fluid with $\mathrm{C}=$ $37.5 \%$ were characterized. The experimental results show that when the ER fluids are free of electric field $(E=0 \mathrm{kV} / \mathrm{mm})$, they are Newtonian. However, for the former two ER fluids, the deposition effect is very remarkable and stirring has to be made continuously to keep the suspension stable. With the increase of the electric field intensity, the yield shear stresses of ER fluids increase exponentially but their viscosities do not change much. It is also found that within the parallel duct, the flow of ER fluids exhibits notable fluctuations, whose period increases with the increase of electric field intensity and is independent of the shear rate.
\end{abstract}

Keywords: Electro-rheological; Bingham plastic; high shear rate; parallel duct; fluctuation.

\section{Introduction}

Electro-rheological (ER) fluid is a smart suspension which consists of large mounts of solid particles (e.g., starch, lime, stone, carbon, etc.) dispensed in a dielectric carrier liquid (such as silicon oil, paraffin, kerosene, etc.) ${ }^{1}$. When the ER fluid is subjected to a high-intensity electric field, it can be changed promptly from Newtonian to Bingham-plastic material and this process is usually within tens millisecond ${ }^{2}$. Besides, the yield shear stress and viscosity of the ER fluids can be controlled by the intensity of the applied electric-filed. Thus, the fast response and controllability of ER fluid make it a promising smart material. Since the development of ER fluid by Winslow ${ }^{3}$ in 1949, ER fluids have been studied extensively in relation to many applications, such as vibration control $^{4-5}$, seismic isolation ${ }^{6}$, clutches ${ }^{7}$ and so on. Moreover, to increase the force of ER dampers, Gavin ${ }^{8}$ and Kuo et al ${ }^{9}$ proposed multi-ducts method to generate high damping 
force. On the other hand, to improve the low yield stress of the previous ER fluids, Wen ${ }^{10}$ developed a new material called giant ER fluid whose yield stress is much higher than the conventional ones.

Although numerous studies have been conducted on various applications of ER fluids, most of them aimed at vibration control within the range of low shear rate, while very few works paid attention to the behaviors of ER fluids under impact scenarios. With the application of ER fluids in adaptive energy absorption in mind, the flowing behavior of ER fluids under high shear rate has attracted our attention. However, the conventional rotational rheometer can only measure the ER fluids under low shear rate $(<1000 / \mathrm{s})$, because for high shear rate, the centrifugal effect of the particles under electric field becomes quite prominent. As reported in this paper, a capillary rheometer with parallel duct has been designed and manufactured, which could measure the properties of ER fluids under a shear rate as high as 5000/s. Utilizing this capillary rheometer, the flowing behaviors of two giant ER fluids with mass faction $55 \%$ and $45 \%$, as well as a density-matched commercial ER Fluid with mass faction $37.5 \%$ were experimentally investigated. The experiments show that the flow of the ER fluid under high electric field is not smooth and fluctuation is quite serious. Based on the experimental results, the relation between the nominal shear stress and the shear rate under different electric fields are obtained.

\section{Preliminary}

In the previous studies, the flowing behavior of ER fluids subjected to high electric field is usually modeled as Bingham plastic with the following constitutive relation

$$
\tau=\tau_{0}+\mu \cdot \dot{\gamma}
$$

where $\tau$ is the shear stress, $\tau_{0}$ is the yield stress, $\mu$ is the viscosity and $\dot{\gamma}$ is the shear rate.

(a)

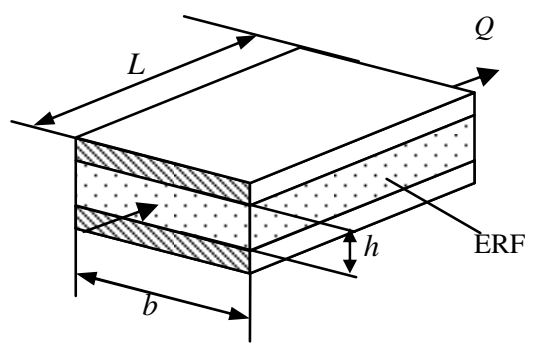

Fig. 1. The flowing behavior of ER fluid: (a) the parallel duct; (b) the cross-section of the duct.

Consider the flowing of the ER fluid through a parallel duct as illustrated in Fig. 1(a), which has length $L$ and width $b$ while the distance between the electrodes is $h$. When the ER fluid is free of electric field, the contour of the stream lines is a parabola S1 as shown in Fig. 1(b). After applying an electric field to the duct, the stream line will change from 
S1 to S2. The flowing in the middle section between A and B is uniform. Then, the relation between the flux of the quasi-steady flow $Q$ and the pressure drop $\Delta P$ through the duct can be obtained from ${ }^{4}$,

$$
\Delta P^{3}-\left(3 \tau_{y} \frac{L}{h}+\frac{12 \mu L Q}{b h^{3}}\right) \Delta P^{2}+4 \tau_{0}^{3}\left(\frac{L}{h}\right)^{3}=0
$$

If the viscosity of the Bingham plastic fluid is known, for given flux $Q$ and the pressure drop $\Delta P$, the yield stress of the ER fluid could be obtained. However, in the present study, both the yield stress and viscosity of the ER fluids need to be determined, so that Eq. (2) alone is not sufficient to determine the yield stress. However, the shear stress along the boundary can be calculated by

$$
\tau=\Delta P \times \frac{h}{2 L}
$$

On the other hand, if the stream-lines are assumed to be similar to those in a Newtonian flow, then the shear rate can be found as follows,

$$
\dot{\gamma}= \pm 6 \frac{u_{m}}{h}= \pm \frac{6 Q}{b h^{2}}
$$

where $\dot{\gamma}$ is the nominal shear rate and $u_{\mathrm{m}}$ is the mean flow speed within the duct. Hence, by employing Eqs. (3) and (4), the shear strain-stress curves of the ER fluids under different electric field can be obtained.

\section{Experimental Setup}

(a)

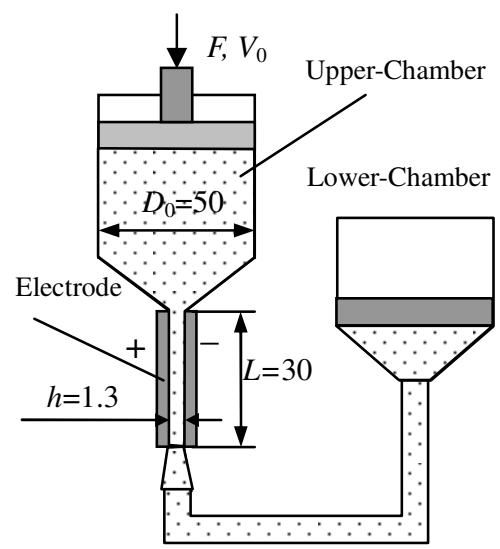

Fig. 2. The design of capillary rheometer for ER fluids.

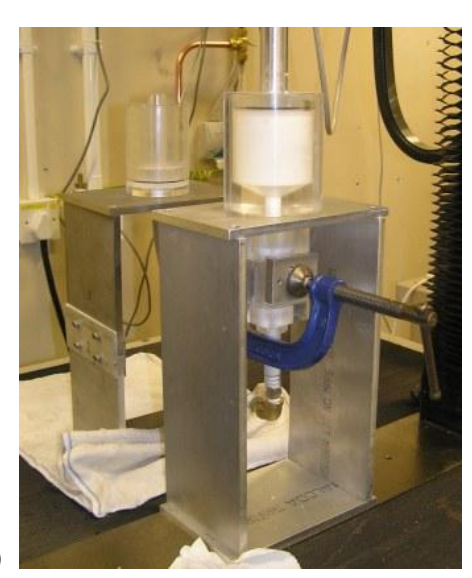

(b)

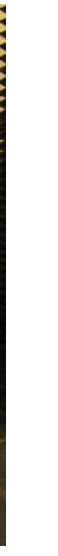

As shown in Fig. 2, the capillary rheometer used in this study is composed of three parts, namely, the upper chamber, the lower chamber and the electro-duct. The chambers are cylindrical with an inner diameter $D_{0}=50 \mathrm{~mm}$, but the duct has a rectangular cross section with $L=30 \mathrm{~mm}, b=10.0 \mathrm{~mm}$ and $h=1.3 \mathrm{~mm}$. The three parts are connected by means of conic transition sections to minimize the pressure loss. In the experiments, the assembled rheometer was placed on an MTS machine. Initially, the ER fluid was sealed inside the chambers and pipes. The piston of the upper chamber was compressed by the MTS 
crosshead with a certain velocity, while the piston of the lower chamber was free. The resistant force of the piston, $F$, coming from four mechanisms, is estimated as

$$
F=\Delta P_{0} \times A_{0}=\left(\Delta P_{f}+\Delta P_{d}+\Delta P_{c}+\Delta P_{g}\right) \times A_{0}
$$

where $\Delta P_{\mathrm{f}}$ is caused by the friction between the pistons and chamber walls, $\Delta P_{\mathrm{d}}$ and $\Delta P_{\mathrm{c}}$ are the pressure drops within the electric duct and the other pipes, respectively, $\Delta P_{\mathrm{g}}$ is resulted from the gravity, and $A_{0}$ is the cross-sectional area of the chambers.

Since this rheometer is designed to test the flowing behavior of ER fluids within the electric duct, the influences of other sections are undesirable. In order to evaluate the effect of the factors such as pipes and gravity, an FEM simulation was conducted by using the given dimensions and assuming viscosity $\mu=0.5$. It is found from the simulation that the pressure loss due to the pipes system is less than $2 \%$ of the total pressure drop. Therefore, in the subsequent analysis, the influences of the pipes and gravity are ignored.

Before the characterization of ER fluids, the rheometer was calibrated using glycerol with mass concentration around $\mathrm{C}>99.0 \%$ under room temperature $20^{\circ} \mathrm{C}$. It was found that except the initial stage, the designed rheometer could obtain very smooth loading curves. Using Eqs. (3) and (4), the shear rates and shear stresses are calculated and the measured viscosity of the glycerine is $1.36 \mathrm{~Pa}$.s. Compared with the standard viscosity value $1.41 \mathrm{~Pa}$.s with $\mathrm{C}=100 \%$, the accuracy of this rheometer is within $5 \%$.

\section{Results and Discussion}

In the experiments, two giant ER fluids produced in the smart material lab at HKUST and a commercial ER fluid were tested, which are denoted by ERF No. 1-3. The giant ER fluids are composed of silicon oil and nano-coated particles ${ }^{10}$, and their mass concentrations are $\mathrm{C}=51 \%$ and $44.5 \%$, respectively. The commercial ER, which consists of silicone oil and particles of Lithium salt and chloro-fluoro polymer, is a density-matched suspension with $\mathrm{C}=37.5 \%$. To characterize the $\mathrm{ER}$ fluids under high intensity electric field, the loading speed of the MTS machine was taken as $V=10-450 \mathrm{~mm} / \mathrm{min}$, and the applied electric field ranged from $E=0 \mathrm{kv} / \mathrm{mm}$ to $3.0 \mathrm{kV} / \mathrm{mm}$. In trial tests, it was found for the giant ER fluids, the deposit effect of the particles was very serious, which made the properties of the ER fluids rather unstable. Therefore, to ensure the experiments repeatable, the ER fluid was stirred to uniform before every test.

The characteristic curves for ERF-1 under zero electric field are plotted in Fig. 3(a). It is shown that loading was stable, and with the increase of the loading speed, the load $F$ also increased. By using Eqs. (3) and (4), the relations between the shear stress and shear rate of the three ER fluids under zero electric field are obtained and depicted in Fig. 3(b). It is shown that the shear stress has a linear relationship with the shear rate, implying that when free of electric field, the ER fluids behave as Newtonian fluids. It is noted that in Fig. 3(b) the intersections of the fitting lines and the $y$-axis are not zero, because of the friction. As shown in Fig. 3(b), the viscosities of the three ERFs under zero electric field are $\mu=0.48$ Pas, 0.10Pas and 0.16Pas, respectively.

In the tests under high-intensity electric field, the piston of the upper chamber moved downwards first, then at a certain moment the electric field was applied. Some typical loading curves are shown in Fig. 4. It can be seen that when the electric field was applied, 
the resistant force increased sharply, since the ERF was changed from Newtonian to Bingham plastic. When subjected to electric field, the loading curves were no longer smooth and exhibited severe fluctuations. It is also found in Fig. 4(a) that for the giant ERF with $\mathrm{C}=51 \%$, when the loading speed was lower than $200 \mathrm{~mm} / \mathrm{min}$, the fluctuation was quite serious. When $V>200 \mathrm{~mm} / \mathrm{min}$, the loading becomes stable. Fig. 4(c) shows that the low density giant ERF have more serious fluctuations, and with the same electric field intensity, lower shear rate will cause fluctuations of larger amplitude. Besides, it is shown in Figs. 4(b) and (d) that for higher electric field, the fluctuation period is shorter and the amplitude is larger. A possible explanation of this phenomenon is that for the flowing of ERF in the parallel duct, the particles near the boundary are subjected to larger shear stress than those in the middle, so that the some particles are captured by the electrodes. With the progress of flowing, more and more particles aggregate near the boundary, resulting in an increase of the resistance to the flowing. When the pressure reaches a critical value, the aggregated particles will be pushed away and the pressure drops soon. The repeat of these processes results in the fluctuations as observed.

The characteristic curves for ERF-1 under zero electric field are plotted in Fig. 3(a). It is shown that loading was stable, and with the increase of the loading speed, the load $F$ also increased. By using Eqs. (3) and (4), the relations between the shear stress and shear rate of the three ER fluids under zero electric field are obtained and depicted in Fig. 3(b). It is shown that the shear stress has a linear relationship with the shear rate, implying that when free of electric field, the ER fluids behave as Newtonian fluids. It is noted that in Fig. 3(b) the intersections of the fitting lines and the $y$-axis are not zero, because of the friction. As shown in Fig. 3(b), the viscosities of the three ERFs under zero electric field are $\mu=0.48$ Pas, 0.10 Pas and 0.16 Pas, respectively.

In the tests under high-intensity electric field, the piston of the upper chamber moved downwards first, then at a certain moment the electric field was applied. Some typical loading curves are shown in Fig. 4. It can be seen that when the electric field was applied, the resistant force increased sharply, since the ERF was changed from Newtonian to Bingham plastic. When subjected to electric field, the loading curves were no longer smooth and exhibited severe fluctuations. It is also found in Fig. 4(a) that for the giant ERF with $\mathrm{C}=51 \%$, when the loading speed was lower than $200 \mathrm{~mm} / \mathrm{min}$, the fluctuation was quite serious. When $V>200 \mathrm{~mm} / \mathrm{min}$, the loading becomes stable. Fig. 4(c) shows that the low density giant ERF have more serious fluctuations, and with the same electric field intensity, lower shear rate will cause fluctuations of larger amplitude. Besides, it is shown in Figs. 4(b) and (d) that for higher electric field, the fluctuation period is shorter and the amplitude is larger. A possible explanation of this phenomenon is that for the flowing of ERF in the parallel duct, the particles near the boundary are subjected to larger shear stress than those in the middle, so that the some particles are captured by the electrodes. With the progress of flowing, more and more particles aggregate near the boundary, resulting in an increase of the resistance to the flowing. When the pressure reaches a critical value, the aggregated particles will be pushed away and the pressure drops soon. The repeat of these processes results in the fluctuations as observed. 


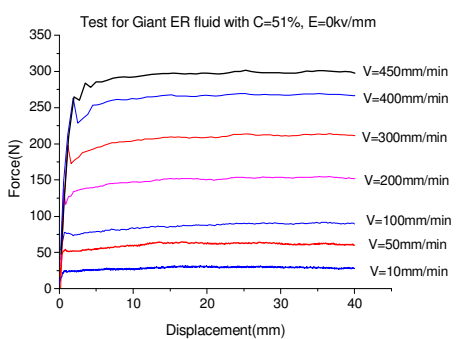

(a)

Fig. 3. Test for the viscosity of the ERFs under zero electric field.

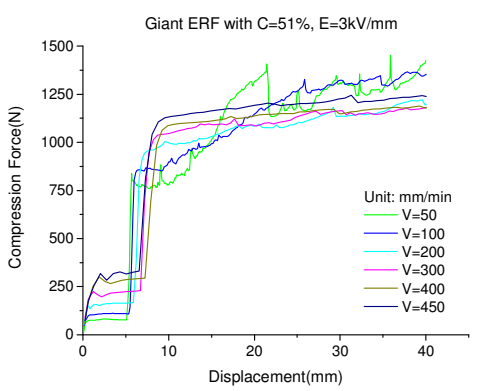

(a)

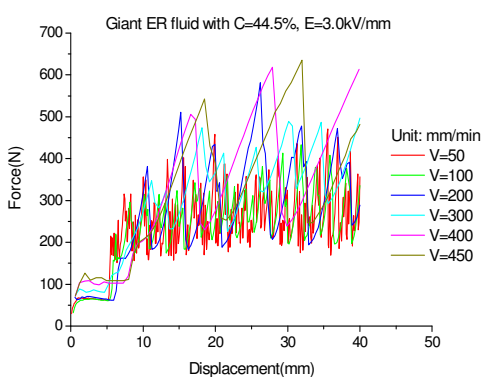

(c)

Fig. 4. Typical loading curves of the ERFs.

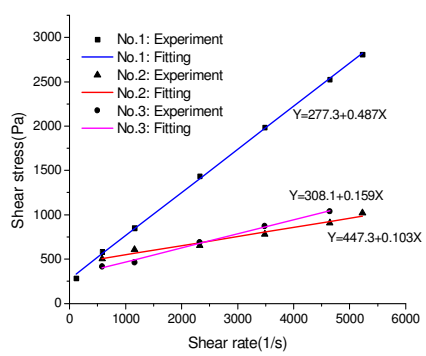

(b)

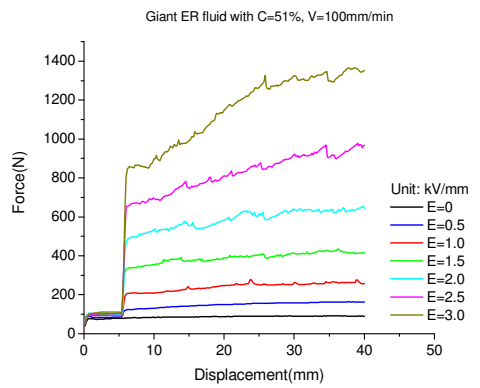

(b)

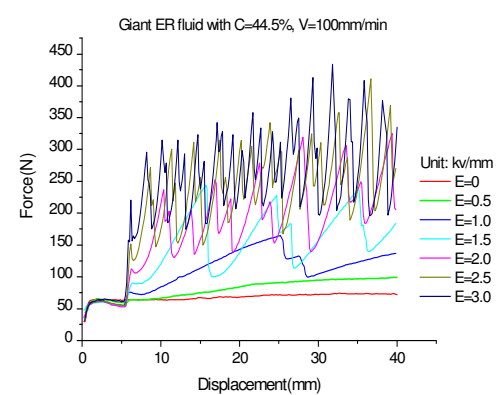



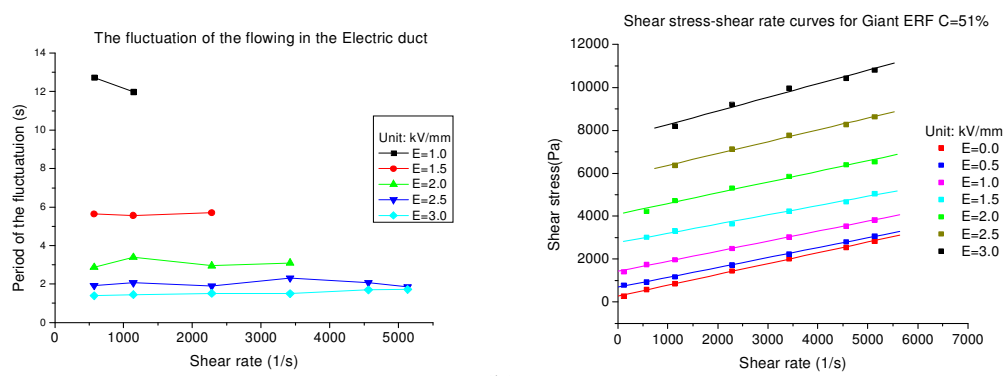

(a)

(b)
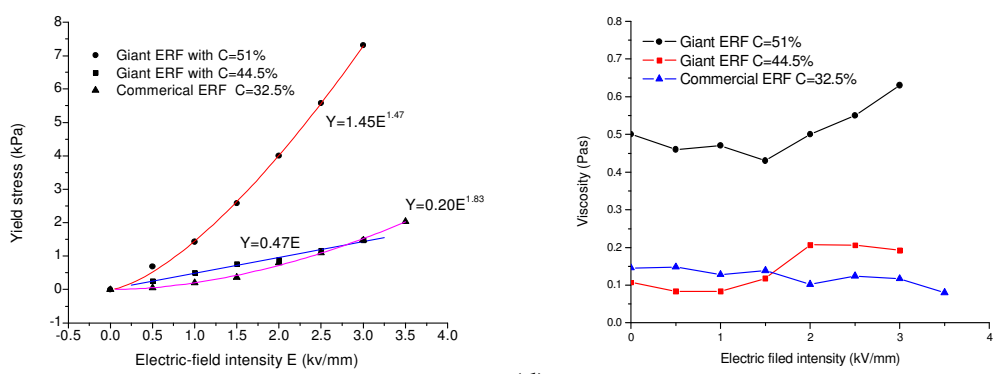

(c)

(d)

Fig. 5. Characterization results of ERFs.

\section{Summary}

In this study, a capillary rheometer has been designed, manufactured and then used to characterize three ERFs under high shear rate within parallel duct. When free of electric field, the ERFs behave like Newtonian fluids; and when subjected to high electric field they are changed to be Bingham plastic. However, the flowing of the ERFs in parallel duct is not smooth and has severe fluctuations, and the period of the fluctuations only depends on the electric field intensity.

\section{Acknowledgment}

The work reported in this paper is part of a project CERG No.621505 supported by the Hong Kong Research Council. Its financial assistance is gratefully acknowledged.

\section{References}

1. R. Stanway, Materials Science and Technology, 20, 931 (2008).

2. V. M. Zamudio, et al, Physica A, 227, 55 (1996).

3. W. M. Winslow, Journal of Applied Physics, 20, 1137 (1949).

4. R. Stanway, et al, Smart Materials and Structures. 5, 464 (1995).

5. G. M. Kamath, et al, Smart Materials and Structures, 5, 576 (1996).

6. N Makris, et al,Smart Materials and Structures, 5, 551 (1996).

7. K. P. Tan, et al, International Journal of Modern Physics B, 20(9), 1049 (2006). 
6036 X. Zhang, C. Zhang \& T. Yu

8. H. P. Gavin, Smart Materials and structures, 7, 664 (1998).

9. W. H. Kuo, et al, Journal of sound and vibration, 292, 694 (2006).

10. W. J. Wen, et al, Nature Materials, 2, 727 (2003). 